\title{
PREPARATION AND CHARACTERISTICS OF NOVEL HYPERBRANCHED PEU-BASED GEL POLYMER ELECTROLYTES
}

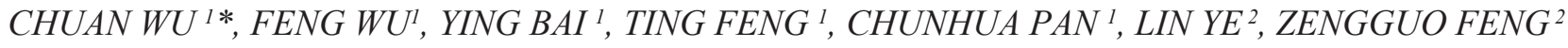 \\ 1. School of Chemical Engineering and the Environment, Beijing Institute of Technology, Beijing 100081, China \\ 2. School of Materials Science and Engineering, Beijing Institute of Technology, Beijing 100081, China
}

(Received: July 30, 2008 - Accepted: May 18, 2009)

\begin{abstract}
Gel polymer electrolytes (GPEs) based on hyperbranched poly (ether urethane) (PHEU) were prepared from the reaction of the hyperbranched polyether (PHEMO) with different diisocyanates in liquid electrolyte. The ionic conductivities and transference number of the GPEs were investigated by alternating current (AC) impedance and multi-potential steps. It is found that the ionic conductivities of GPEs were influenced by diisocyanate structure, lithium salt concentration and addition amount of liquid electrolyte. Sample PUM- $0.5 \mathrm{M}-80 \%$ originated from 4, 4-diphenylmethane diisocyanate (MDI) as the hard segment shows the highest ionic conductivity of $2.41 \times 10^{-3} \mathrm{~S} \cdot \mathrm{cm}^{-1}$ at ambient temperature. The lithium ion transference number is 0.7 . In addition, small molecules of liquid electrolyte were perfectly entrapped by polymer matrix, and liquid leakage in lithium ion battery was avoided.
\end{abstract}

Keywords: Hyperbranched PEU; GPE; Ionic conductivity; lithium ion battery

\section{INTRODUCTION}

GPEs are paid more and more attentions to because of their applications to lithium ion batteries in recent years ${ }^{1}$. However, for the conventional poly (ethylene oxide) (PEO)-LiX polymer electrolyte, the significant drawback is the low conductivity at ambient temperature, which is due to high crystallization and poor motion performance of polymer host ${ }^{2-4}$. Therefore, there is a continuous challenge to search for a novel polymer electrolyte with high ionic conductivity.

Poly (ether urethane) (PEU) has a two-phase microstructure, where the hard segment domains are distributed in a soft segment matrix ${ }^{5}$. The hard segment provides good mechanical strength, while the soft segment improves the ionic conductivity. The rubbery soft segments can dissolve alkali metal salts, and help achieving high ion mobility, which originates from the interaction of the ether oxygen with the alkali metal ions. In the previous report ${ }^{6}$, the hyperbranched polyether (PHEMO) without stiff groups is selected as soft segment, as well as its amorphous state inherently derived from the hyperbranched molecular structure. Therefore, PHEMO is expected to improve the ionic conductivity of polymer electrolyte ${ }^{7}$. Due to their good mechanical properties, diisocyanates are chosen to act as hard segment domains contributing to the dimensional stability of the polymer electrolytes ${ }^{8}$.

In this paper, GPEs based on PHEU were prepared with hyperbranched polyether (PHEMO) and diisocyanates in liquid electrolyte. Diisocyanates with different structures, such as aliphatic isocyanates of hexamethylene diisocyanate (HDI) and isophorone diisocyanate (IPDI), or aromatic isocyanates of 4, 4-diphenylmethane diisocyanate (MDI) and 2, 4-tolune diisocyanate (TDI) were served as hard segments, respectively. The effects of diisocyanate structure, lithium salt concentration and addition amount of electrolyte on the ionic conductivities of the as-prepared GPEs were discussed and compared.

\section{EXPERIMENTAL}

\section{Materials}

Lithium trifluoromethanesulfonimide (LiTFSI) was purchased form Aldrich. The other reagents used to synthesize PHEMO were described in our previous work ${ }^{[6]}$. The diisocyanate, i.e., HDI, MDI, IPDI, and TDI were kindly provided by Liming Institute of Chemical Industries, China. Triethylene glycol dimethyl ether (TGE) and dibutyltin dilaurate (T-12) were purchased from Acros.

Preparation of GPE

The synthesis of PHEMO has been described previously ${ }^{6,9}$. A series of TGE-dissolved LiTFSI with various concentrations from 0.1 to $3.0 \mathrm{~mol} / \mathrm{L}$ were prepared, and used as the liquid electrolytes. Different amount of PHEMO was mixed with diisocyanate in the liquid electrolyte, and then T-12 catalyst was added. This reaction was carried out in a nitrogen-flushed oven at $50 \square$ for $24 \mathrm{hrs}$ to obtain a PHEU gel polymer electrolyte. Different GPEs were obtained by using various diisocyanates. Fig.1(a) shows the scheme of the preparation of PHEU, and the structures of HDI, MDI, IPDI and TDI are shown in Fig.1(b).

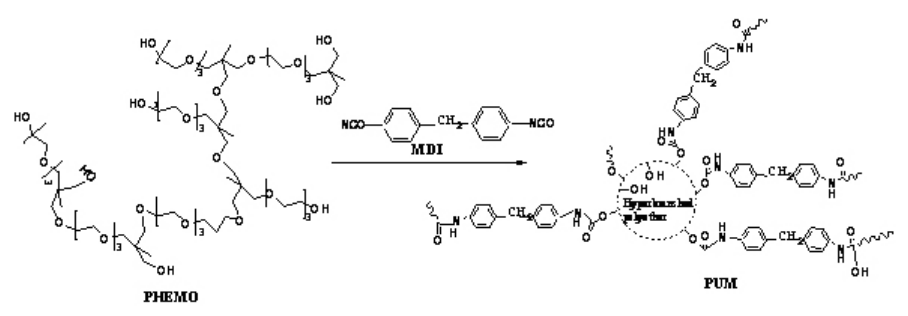

(a)<smiles>[O][N+]([O-])CCCCCCC[N+](=O)[O-]</smiles>

(1) HDI

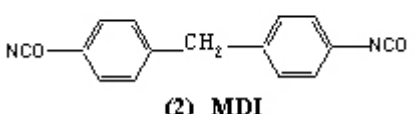

(2) MDI

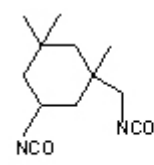

(3) IPDI

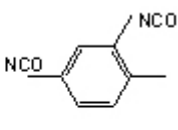

(4) TDI (b)

Fig.1 Scheme of the preparation of PHEU by the reaction of PHEMO and MDI (a); the structure of HDI, MDI, IPDI and TDI (b)

\section{Composition of GPE}

In this study, PUH, PUM, PUI and PUT represent the as-prepared GPEs, where the hard segments of the polymer matrix are HDI, MDI, IPDI and TDI, respectively. PUH-1M-50\% means the GPE with the hard segment of HDI, the lithium salt concentration of $1 \mathrm{~mol} / \mathrm{L}$ and the addition amount of liquid electrolyte of $50 \%$ ( $50 \%$ refers to the mass ratio of the liquid electrolyte to the polymer matrix).

Electrochemical measurements

The ion conductivities were evaluated from A.C. impedance technique over a frequency range $1 \square 10^{5} \mathrm{~Hz}$ using $\mathrm{CHI} 660 \mathrm{~A}$ electrochemistry workstation (Shanghai Chenhua Instrument Co. Ltd.). The measurements were carried out from 30 to $80 \square$ with an interval of $10 \square$.

Lithium ion transference number, $t_{\mathrm{Li}}$, was tested using the method of multi-potential steps, which was calculated using the following equation: $t_{\mathrm{Li}}$ $=I \mathrm{ss} \square I_{0}$, where $I_{0}$ and $I_{\mathrm{ss}}$ are the initial and steady-state currents, respectively ${ }^{10}$. A well sealed symmetrical $\mathrm{Li} / \mathrm{GPE} / \mathrm{Li}$ experimental cell was assembled in a glove box and tested using $\mathrm{CHI} 660 \mathrm{~A}$ electrochemistry workstation to determine the transference number $t_{\mathrm{Li}+}$.

\section{RESULTS AND DISCUSSION}

\section{Ionic conductivities}

The ionic conductivities of the as-prepared GPEs have a tendency to increase with the increasing temperature, following the Arrhenius equation ${ }^{11}$, as shown in Fig.2. With increasing temperature, the mobility of polymer chains 
is enhanced, and the fraction of free volume in the polymer matrix increases. Therefore, high ionic conductivity can be achieved. With the same lithium salt concentration and the same electrolyte adding amount, PUH and PUM with straight chain deliver higher ionic conductivities than PUI and PUT with ring structure, because straight chains can achieve excellent flexibility and mobility 12,13

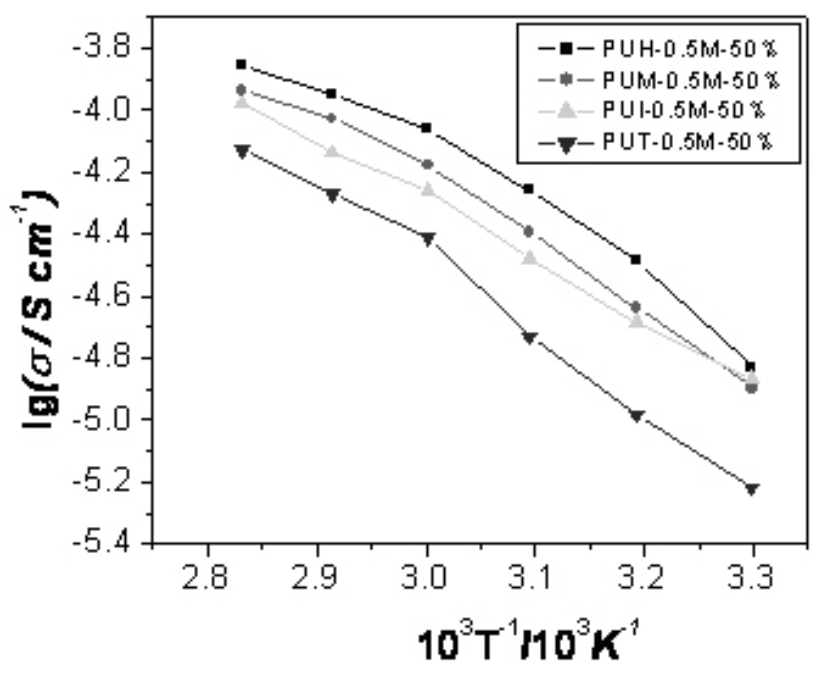

Fig. 2 Dependence of ionic conductivities on different diisocyanates

Fig.3 shows the dependence of the ionic conductivities on different lithium salt concentration. Four curves show a trend of increase at first, and then decrease. It is observed that when the lithium salt concentration is $1 \mathrm{~mol} / \mathrm{L}$, PUH and PUM have the highest conductivities at ambient temperature, whereas PUI and PUT have the highest conductivities with $2 \mathrm{~mol} / \mathrm{L}$ lithium salt. It is well known that the amount of lithium ion plays an important role on the ion conductive process. With increasing lithium salt concentration, the amount of free transfer lithium ion increases correspondingly, therefore leads to an ionic conductivity enhancement. However, when increasing lithium salt concentration to some degree, formation of transient cross-link between lithium ion and the ether group can decrease the segmental motion of the polymer chain ${ }^{14}$, which results in a decrease in the mobility of the charge carrier.

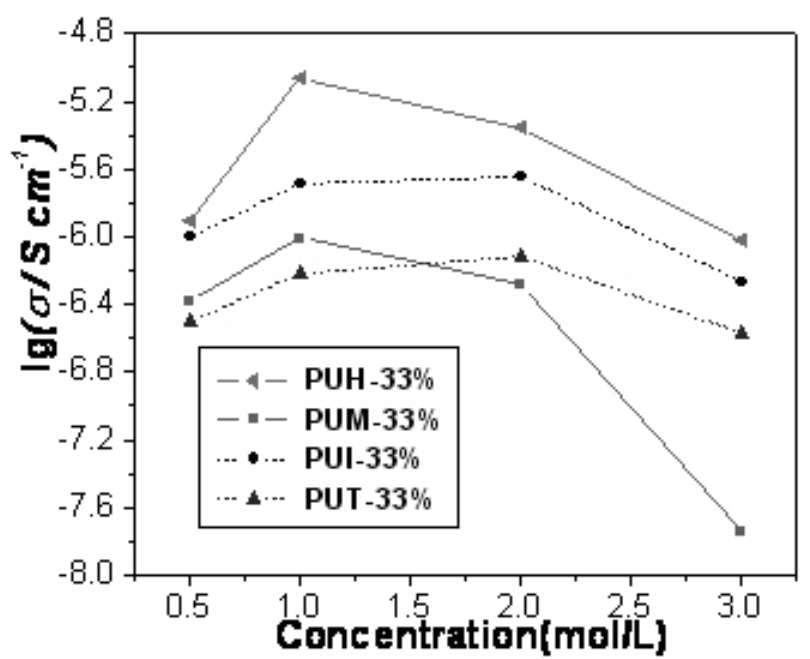

Fig. 3 Dependence of ionic conductivities on different lithium salt concentrations

Fig. 4 shows the relationship between the conductivities of the GPEs and the adding amounts of the liquid electrolytes. When the lithium salt concentration remains constant, the ionic conductivities of PUH, PUM, PUI and PUT strongly increase with the increasing addition amount of the liquid electrolyte. As we known, the ionic conductivity is mainly dependent on the diffusion rate and the amount of lithium ion. Therefore, the more the adding amount of the liquid electrolyte is, the more the content of lithium ion is. The GPE is composed of polymer matrix containing the liquid electrolyte, which is similar to a stuffing enclosed with a piece of dumpling wrapper. If there is too much stuffing, the dumpling wrapper may be broken. Namely, if the addition amount of the liquid electrolyte is too much to be involved in the polymer matrix, the GPE fails to form a free-standing membrane. Therefore, in this study, the maximum adding amount of liquid electrolyte for PUH, PUI and PUT is proved to be $67 \mathrm{wt} \%$, whereas that for PUM is $80 \mathrm{wt} \%$. It can be found that when the lithium salt concentration is $0.5 \mathrm{~mol} / \mathrm{L}$ and the GPE containing $67 \mathrm{wt} \%$ liquid electrolyte, the conductivities of PUH, PUM, PUI and PUT are $3.52 \times 10^{-4} \mathrm{~S} \cdot \mathrm{cm}^{-1}, 4.25 \times 10^{-4} \mathrm{~S}^{\circ} \mathrm{cm}^{-1}, 4.74 \times 10^{-4} \mathrm{~S} \cdot \mathrm{cm}^{-1}, 2.59 \times 10^{-4}$ $\mathrm{S} \cdot \mathrm{cm}^{-1}$ at ambient temperature, respectively. Moreover, it is clear that the ionic conductivity of PUM- $0.5 \mathrm{M}-80 \%$ containing $80 \mathrm{wt} \%$ liquid electrolyte is the highest, $2.41 \times 10^{-3} \mathrm{~S} \bullet \mathrm{cm}^{-1}$ at ambient temperature, as shown in Fig.4(b). Because the hard segment of MDI has a straight chain with two phenyls, PUM exhibits better mechanical properties than the other GPEs do ${ }^{15}$, therefore it can entrap more liquid electrolytes, and result in the highest ionic conductivity.
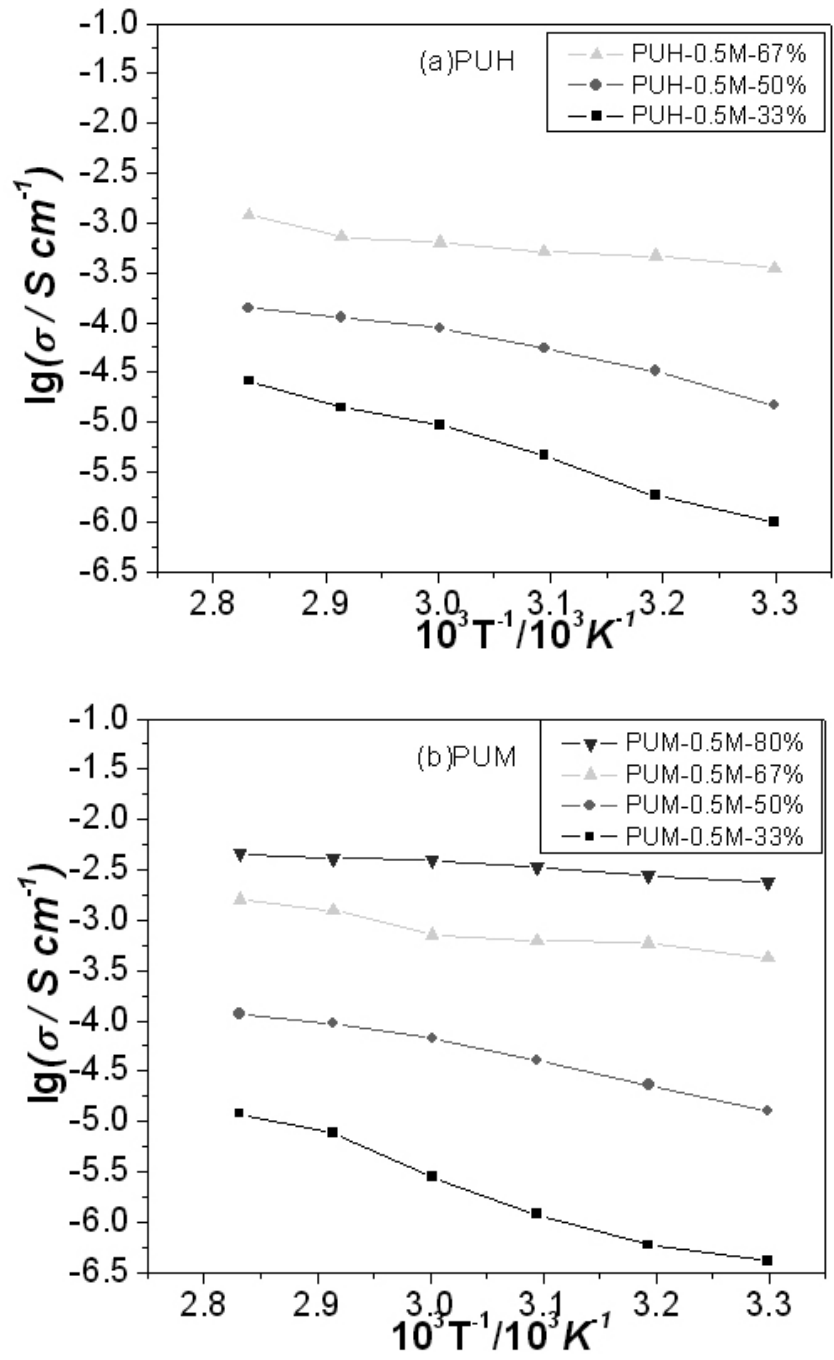

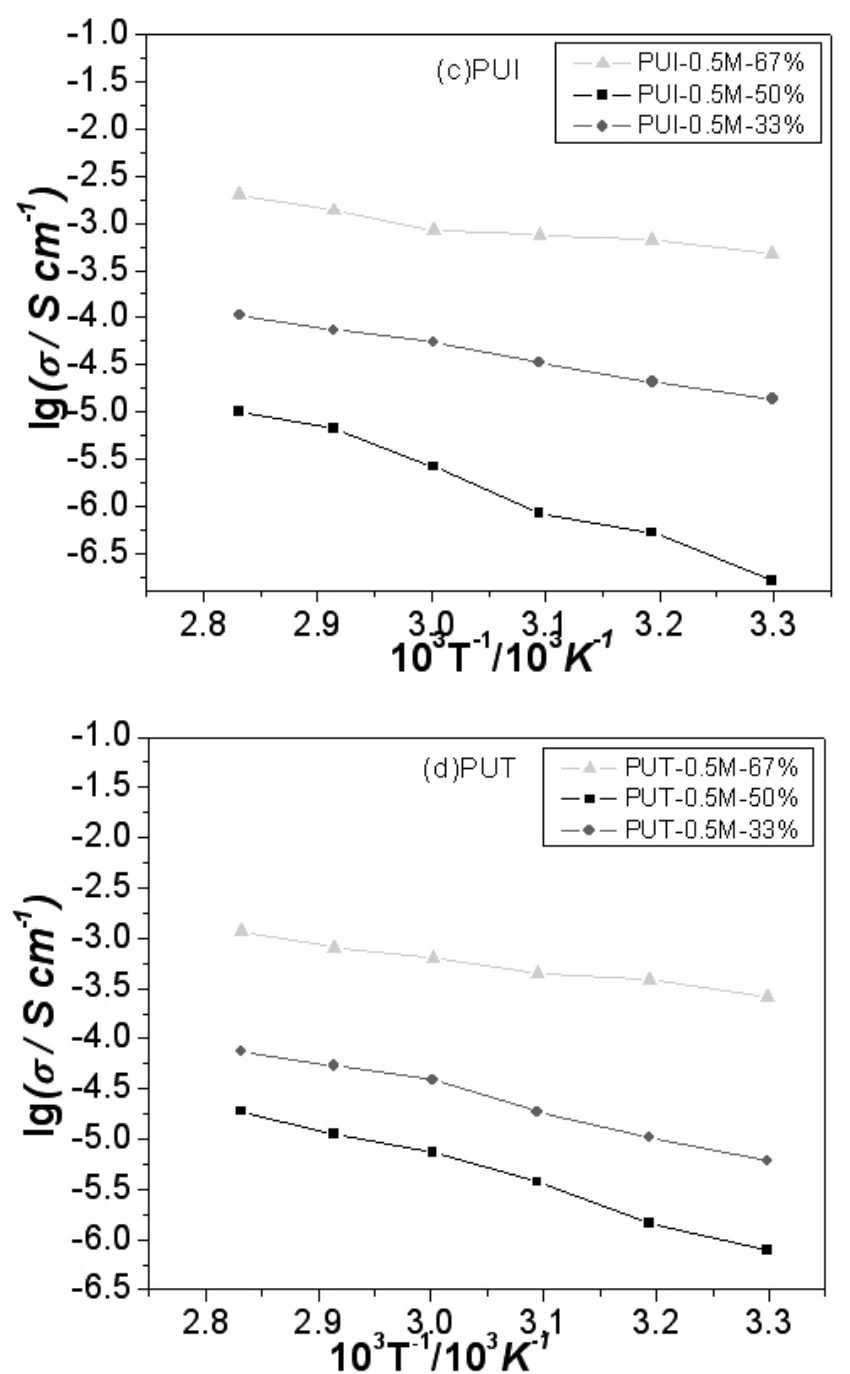

Fig. 4 Dependence of ionicconductivities on the adding amount of the liquid electrolytes.

\section{Lithium ion transference number}

Lithium ion transference number, $t_{\mathrm{Lit}}$, which is one of the most important parameters for lithium ion batteries, was evaluated using multi-potential steps technique. A relative high $t_{\mathrm{Li}+}$ can eliminate the concentration gradients in the battery and ensure its operation at high current density. All parameters used for the calculation of $t_{\mathrm{Li}+}$ can be obtained directly from the current response of the $\mathrm{Li} / \mathrm{GPE} / \mathrm{Li}$ experimental cell. In the system of PUM- $0.5 \mathrm{M}-80 \% \mathrm{GPE}$, lithium ion transference number is as high as 0.7 , as shown in Fig.5.

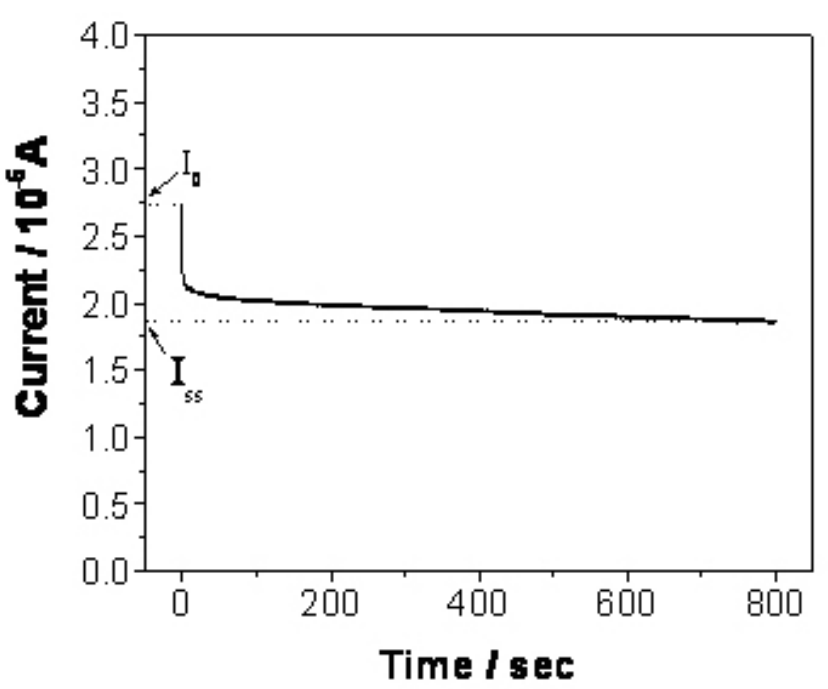

Fig.5 Multi-potential steps curves of PUM-0.5M-80\% GPE

\section{CONCLUSIONS}

A novel type of hyperbranched gel polymer electrolytes is prepared by PHEMO and different diisocyanates. Ionic conductivities of the as-prepared gel polymer electrolytes increase at first, and then decrease with the increasing lithium salt concentrations. The amount and mobility of the lithium ion can be enhanced by increasing the adding amount of the liquid electrolyte. The highest ionic conductivity is $2.41 \times 10^{-3} \mathrm{~S} \cdot \mathrm{cm}^{-1}$ (sample PUM- $0.5 \mathrm{M}-80 \%$ ) at ambient temperature, because PUM with the MDI consisting of a chain with two phenyls, therefore this GPE has better mechanical properties and can entrap more liquid electrolytes. In addition, the small molecules of the liquid electrolyte were perfectly enwrapped by the polymer matrix, thus help avoiding the electrolyte leakage, and enhancing the safety of lithium ion battery.

\section{ACKNOWLEDGEMENTS}

The present work is financially supported by the National Natural Science Foundation of China (grant No. 20703005), the National 973 Program (grant No. 2009CB220100), Teaching \& Researching Fund of BIT (grant No. 20060542012), and Beijing Excellent Talent Support Program (grant No. 20071D1600300396)

\section{REFERENCES}

1.- F. R. Kalhammer. Solid State Ionics 135, 315, (2000)

2.- J. Vondrák, J. Reiter, J. Velická, M. Sedlaříková. Solid State Ionics 170 , 79, (2004)

3.- D. E. Fenton, J. M. Parker, P. V. Wright. Polymer 14, 589, (1973)

4.- M. B. Armand, M. Duclo. Eur. Patent 0013199, 1978

5.- M. Digar, S. L. Hung, H. L. Wang, T. C. Wen, A. Gopalan. Polymer 43, 681, (2002)

6.- L. Ye, Z. G. Feng, Y. M. Zhao, F. Wu, S. Chen, G. Q. Wang. J. Polym. Sci. 44, 3650, (2006)

7.- F. Wu, T. Feng, Y. Bai, C. Wu, L. Ye, Z. G. Feng. Solid State Ionics 180 677, (2009)

8.- T. C. Wen, Y. L. Du, M. Digar. Eur. Polym. J. 38, 1039, (2002)

9.- Y. Bai, C. H. Pan, F. Wu, C. Wu, L. Ye, Z. G. Feng, Chem. J. Chinese U. 28, 1796, (2007)

10.- J. Y. Xi, X. Z. Tang. Chem. Phys. Lett. 400, 68, (2004)

11.- B. Yang, W. H. Zhu, X. L. Wang, X. Z. Tang, P. F. Zhu, Eur. Polym. J. 37 1813, (2001)

12.- C. H. Pan, Y. Bai, F. Wu, L. Ye, C. Wu, Z. G. Feng. J. Funct. Mater. 38 210, (2007)

13.- L. Ye, Q. Qin, Z.G. Feng, X. W. Zhang, Y. Bai, F. Wu, Polym. Int. 56, 660, (2007)

14.- T. C. Wen, H. H. Kuo, A. Gopalan. Solid State Ionics, 147, 171, (2002)

15.- F. C. Xie, C. C. Zhu, Y. Q. Zhang. China Plast. Ind. 33, 44, (2005). 